\title{
Microscopy Techniques and Technologies Used in Ultrastructural Analyses of Poliovirus Replication Complexes Formed During Infection of HeLa Cells
}

\author{
ER Fischer*, V Nair*, BT Hansen*, FH Hoyt**, G. Belov***, E. Ehrenfeld*** \\ *NIH/NIAID/RML/ RTB/EM Unit, 903 South $4{ }^{\text {th }}$ Street, Hamilton, Montana 59840 \\ **Montana State University, 200 North Hedges Bozeman, MT 59715 \\ ***NIH/NIAID/LID 50 South Drive, Bethesda, MD 20892
}

Cells infected with positive-strand RNA viruses undergo an extensive remodeling of their intracellular membranes to form unique structures on which viral genome replication takes place [1]. One of the best studied examples of membrane rearrangement to form functional RNA replication complexes has been poliovirus (PV) infection of HeLa cells, in which clusters of virus-induced vesicle-like structures have been observed by conventional transmission electron microscopy (TEM) [2](Fig. 1 A\&B). In this study, we discuss the techniques and technologies used to examine the replication complexes formed in PV-infected cells to elucidate the structure of the membranous replication complexes.

Immune-labeling sensitive internal antigens prior to embedding allows use of mild fixatives followed by post-fixation steps after immunology for better preservation of membranes compared to many alternative strategies involving post-section labeling techniques [3]. Antibody labeling against viral replication proteins using horseradish peroxidase secondary conjugates and the electron dense DAB substrate demonstrated the involvement of both single- and double-membrane structures in virus replication (Fig. 1C).

High pressure freezing PV infected cells followed by freeze-fracture and viewing under cryoconditions with an in-lens scanning electron microscope revealed small aggregates of vesicles, consistent with those viewed by TEM (Fig. 2A). A contiguous membranous structure appeared to compartmentalize vesicles into discrete clusters (Fig. 2B). Additional immunological and temporal studies of these structures may provide insight in determining a mechanism for replication complex morphogenesis.

Tilt series of replication complexes were collected and three-dimensional reconstructions revealed the vesicles were, in fact, irregularly shaped convoluted chambers, some with tubular extensions, often in close association with "vesicles" of equal complexity (Fig. 3A-C). A combination of these and other molecular and biochemical techniques will be useful in further studies to determine if there are functional differences between the single and double membrane structures, of if they are merely derived from different membranous origins.

References

[1] A. Salonen, T Ahola, and L. Kaariainen, (2005) Curr Top Microbiol Immunol 285, 139-73. 
[2] D. Egger and K. Bienz, J Gen Virol (2005)86(Pt 3): 707-18

[3] WJ Brown, MG Farquhar, Meth. Cell Biol. (1989)(31) 553
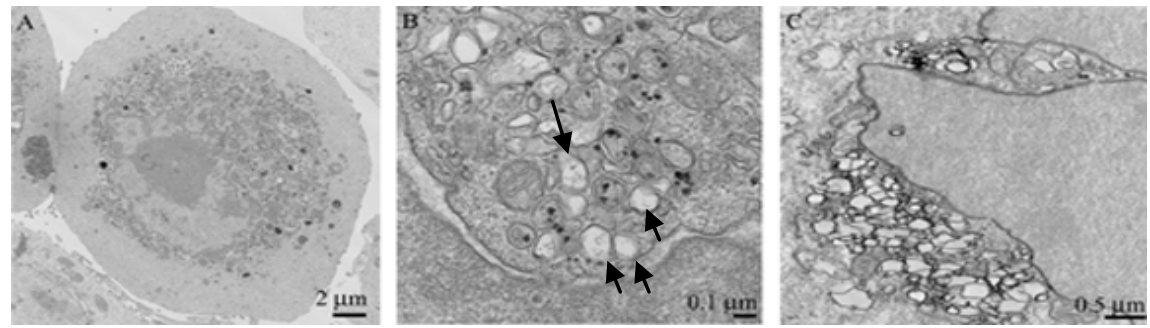

Figure 1. (A) Polioviruses (PV) induce dramatic membrane rearrangement in HeLa cells, (B) to form vesicular shaped replication complexes (arrows), where (C) viral replication occurs as visualized through immunolabeling of viral proteins.
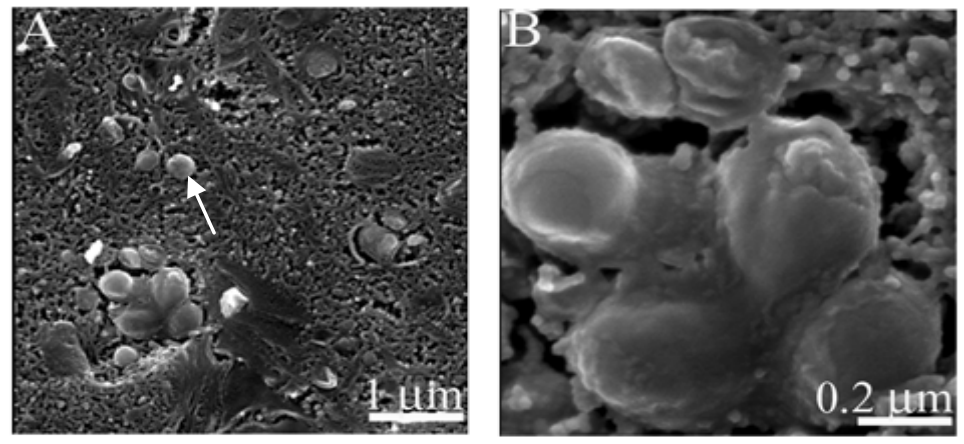

Figure 2. (A) Cryo-SEM of freeze-fractured PV infected Hela cells allows topographical visualization of the viral replication complexes, revealing (B) a membranous structure that appears to encapsulate multiple vesicular structures.
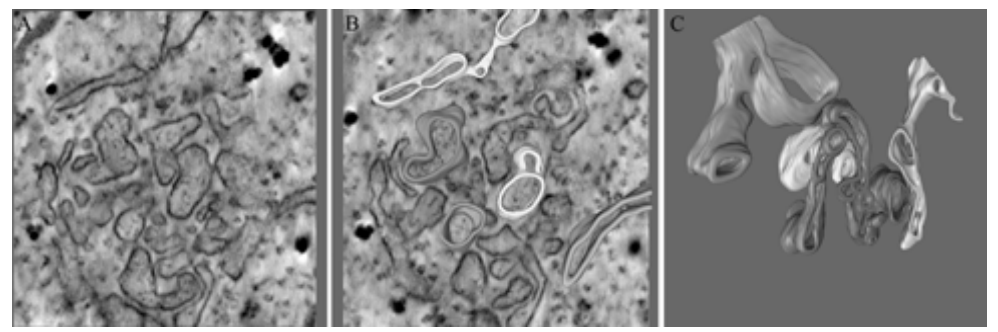

Figure 3. (A) a +/- 70 degree tilt series of a $200 \mathrm{~nm}$ thick region with PV replication associated structures was collected on a FEI Tecnai 120 Biotwin microscope. (B) The final volume was segmented with Amira software (v. 5.2) and (C) the 3-D volume revealed the complexity of membrane compartments involved with viral synthesis. 